DOI: http://dx.doi.org/10.5902/2179-460X18049

Revista do Centro do Ciências Naturais e Exatas - UFSM

Ciência e Natura, Santa Maria,

ISSN: 2179-460X, v.37 n.4 set-dez. 2015, p. 20-27

ciênciaenatura

\title{
Medidas de dependência espacial baseadas em duas perspectivas do semivariograma paramétrico
}

\author{
Spatial dependence measures based on two parametric semivariogram perspectives
}

\author{
Enio Júnior Seidel ${ }^{1}$, Marcelo Silva de Oliveira ${ }^{2}$ \\ Departamento de Estatística - Universidade Federal de Santa Maria, RS, Brasil, (ejrseidel@hotmail.com) \\ 2 Universidade Federal de Lavras, MG, Brasil (marcelo.oliveira@dex.ufla.br)
}

\begin{abstract}
Resumo
Ao se estudar o semivariograma tem-se a possibilidade de calcular medidas descritivas do grau da dependência espacial. Nesse sentido, tem-se o objetivo de apresentar duas medidas de dependência espacial construídas a partir de duas perspectivas geométricas distintas do semivariograma. A primeira medida (denominada de IDE1), baseada no conceito de áreas de dependência espacial observadas no semivariograma. A segunda (denominada de IDE2), com base no conceito de que o semivariograma possui duas áreas distintas, uma área de dependência espacial e uma área de não dependência espacial. O IDE1 é idêntico ao índice DE já utilizado na literatura, ou seja, é possível dizer que encontrou-se, a partir da geometria do semivariograma, a demonstração matemática do índice DE. O IDE2 é mais completo que o indice IDE1, pois considera o fator de modelo, mostrando que há diferenças na dependência espacial dos fenômenos a partir dos modelos que os descrevem. Contudo, esses dois índices não contemplam o alcance prático. Neste sentido, pode-se considerar que as medidas IDE1 e IDE2 podem ser usadas para descrever a dependência espacial, mas devem ser usados em conjunto com outras medidas que contemplem o alcance de dependência espacial.

Palavras-chave: Estatística espacial, Geoestatística, Análise variográfica, Variabilidade espacial, Medidas descritivas.
\end{abstract}

\begin{abstract}
On studying the semivariogram, there is the possibility of estimating descriptive measures of spatial dependence degree. In this study, we aimed to show two spatial dependence measures developed from two distinct geometric perspectives of the semivariogram. The first measure, named IDE1, is based on the concept of spatial dependence areas found in the semivariogram; while the second, named IDE2, is based on the concept that the semivariogram has two distinct areas, an of spatial dependence, and other of non-spatial dependence. The IDE1 is identical to the DE index found in the literature, so that it is possible to state that the mathematical proof of the DE index was from the geometry of semivariogram. The IDE2 is more complete than the IDE1 index because it considers the factor of model, showing that there are differences in spatial dependence of phenomena obtained from their relating models. These two indices do not contemplate the practice reach. Thus, we may consider that the estimates of IDE1 and IDE2 may be used to describe the spatial dependence; however, they should be used along with other measures which contemplate the reach of the spatial dependence.

Keywords: Spatial statistics, Geostatistics, Variographic analysis, Spatial variability, Descriptive measures.
\end{abstract}




\section{Introdução}

Fenômenos que apresentam dependência espacial, em Geoestatística, ao serem modelados atendendo a estacionariedade de $2^{a}$ ordem, tem seus modelos com necessariamente pelo menos quatro parâmetros: contribuição $(C T)$, efeito pepita $(E P)$, patamar $(P T)$ e alcance $(A L)$ (SEIDEL, 2013; SEIDEL; OLIVEIRA, 2014a). Além destes parâmetros de modelo, pode-se também atribuir medidas estatísticas descritivas sobre os dados coletados no fenômeno (que é entendido como uma realização do modelo atribuído) correspondendo aos parâmetros do modelo. Estas medidas descritivas dos dados do fenômeno receberão a designação de parâmetros do fenômeno, para realçar esta correspondência.

Nos modelos teóricos utilizados para descrever tais fenômenos (geralmente modelos esférico, exponencial e gaussiano), tem-se que $o$ parâmetro contribuição é igual à contribuição do fenômeno ( $C_{1}=C T$ ), o efeito pepita, também, é o mesmo do fenômeno ( $C_{0}=E P$ ), e o patamar, por consequência, também é igual ao patamar da dependência espacial do fenômeno $\left(C=C_{0}+C_{1}=P T\right)$ (SEIDEL, 2013).

Contudo, em relação ao parâmetro alcance dos modelos, deve-se fazer uma observação importante. Nos fenômenos em que se ajusta modelo esférico, o alcance real é finito. Já nos fenômenos em que se ajustam modelo exponencial ou gaussiano, o alcance real é infinito. Contudo, para fins de operações matemáticas, convenciona-se que o alcance é virtualmente finito (SEIDEL, 2013; SEIDEL; OLIVEIRA, 2013).

Para o modelo esférico de dependência espacial tem-se que o parâmetro é igual ao alcance prático. Contudo, para o modelo exponencial, tem-se que o parâmetro é igual a $A L / 3=a / 3$. No modelo gaussiano, tem-se o parâmetro $A L / \sqrt{3}=a / \sqrt{3} \quad$ (SEIDEL; OLIVEIRA, 2013; SEIDEL, 2013). Dessa forma, tem-se uma parametrização mais adequada, no sentido de que permite uma interpretação mais facilitada da diferença que existe entre o alcance prático e o parâmetro nos modelos exponencial e gaussiano (SEIDEL, 2013).

O modelo esférico é dado na forma (ISAAKS; SRIVASTAVA, 1989; SEIDEL, 2013):

$\gamma(h)_{e s f}= \begin{cases}0 & , h=0 \\ C_{0}+C_{1}\left[1,5\left(\frac{h}{a}\right)-0,5\left(\frac{h}{a}\right)^{3}\right], 0<h \leq a \cdot(1) & , h>a \\ C_{0}+C_{1} & , 0\end{cases}$

Já o modelo exponencial é dado por (ISAAKS; SRIVASTAVA, 1989; SEIDEL, 2013):

$\gamma(h)_{\exp }=\left\{\begin{array}{l}0 \\ C_{0}+C_{1}\left[1-e^{\left(-\frac{h}{a / 3}\right)}\right], h \neq 0\end{array}\right.$.

O modelo gaussiano é escrito como (ISAAKS; SRIVASTAVA, 1989; SEIDEL, 2013):

$\gamma(h)_{\text {gau }}=\left\{\begin{array}{l}0 \\ C_{0}+C_{1}\left\{1-e^{\left[-\left(\frac{h}{a / \sqrt{3}}\right)^{2}\right]}\right\}, h \neq 0 \\ , h \neq 0\end{array}\right.$

em que $C_{0}$ é o parâmetro efeito pepita, $C_{1}$ é o parâmetro contribuição, $a$ é $\mathrm{o}$ parâmetro alcance, $C_{0}+C_{1}$ é o parâmetro patamar e $h$ é a distância entre pontos. Nos casos de ausência de dependência espacial, ajusta-se o modelo de efeito pepita puro.

Nos modelos exponencial e gaussiano, o alcance $A L$ (alcance prático) é obtido em 95\% da contribuição (BIONDI; MYERS; AVERY, 1994; SEIDEL, 2013).

Ao se considerar o semivariograma e o ajuste de um dos modelos teóricos, tem-se a possibilidade de calcular uma medida de permita descrever o grau da dependência espacial. 
Uma das medidas mais utilizadas para descrever tal grau da dependência espacial é dada por (BIONDI; MYERS; AVERY, 1994):

$$
D E(\%)=\left(\frac{C_{1}}{C_{0}+C_{1}}\right) \cdot 100
$$

em que $C_{0}$ é o efeito pepita e $C_{1}$ é a contribuição.

Esta medida assume valores no intervalo entre zero e $100 \%$. Quanto mais próxima de 100\%, maior é o grau da dependência espacial. $\mathrm{O}$ fato de avaliar o grau da dependência é importante para se comparar fenômenos (diferentes cenários de dependência espacial) (BIONDI; MYERS; AVERY, 1994).

Em Cambardella et al. (1994) é apresentada uma medida semelhante a anterior, podendo ser entendida como sendo o seu inverso, mas tendo, em essência, a mesma possibilidade de interpretar sobre o grau da dependência espacial.

Partindo do conceito de correlação espacial em Geoestatística, Seidel e Oliveira (2014a) apresentaram uma nova medida do grau de dependência espacial, denominada de índice de dependência espacial (IDE), dada pela expressão:

$$
\operatorname{IDE}(\%)=F M \cdot\left(\frac{C_{1}}{C_{0}+C_{1}}\right) \cdot\left(\frac{a}{q \cdot M D}\right) \cdot 100,
$$

em que $F M$ é o fator de modelo (no caso esférico, $\mathrm{FM}=0,375 ;$ no caso exponencial, $\mathrm{FM}=0,317$; e no caso gaussiano, $\mathrm{FM}=0,504), C_{0}$ é o efeito pepita, $C_{1}$ é a contribuição, $a$ é o alcance prático e q.MD é o valor que corresponde à proporção $(q)$ que se deseja atingir da máxima distância ( $M D$ ) entre pontos amostrados. Em sua validação, Seidel e Oliveira (2014a) utilizaram $q=0,5$ para se atingir metade da máxima distância entre pontos amostrados.

Este índice varia no intervalo de zero até $F M .100 \%$ (no caso esférico, varia de 0 a $37,5 \%$; no caso exponencial, varia de 0 a $31,7 \%$; e no caso gaussiano, varia no intervalo de 0 a 50,4\%) (SEIDEL; OLIVEIRA, 2014a). Ainda, de acordo com Seidel e Oliveira (2014a) esta medida é mais completa, pois contempla, em sua essência, uma medida de correlação espacial, além de considerar todos os parâmetros dos modelos da estrutura de dependência espacial.

A etapa de descrição e avaliação da dependência espacial, ao se estudar o semivariograma (estimação das semivariâncias e ajuste de modelo), é fundamental para a realização de uma estimativa adequada, pelo método de krigagem, da variável em estudo em locais não amostrados (CARVALHO; SILIVEIRA; VIEIRA, 2002; OLIVEIRA et al., 2006; SILVA et al., 2010).

Nesse sentido, tem-se o objetivo, neste estudo, de apresentar medidas de dependência espacial construídas a partir de duas perspectivas geométricas distintas do semivariograma.

\section{Metodologia}

Propõe-se a construção de duas medidas descritivas da dependência espacial.

A primeira é baseada no conceito de áreas de dependência espacial observadas no semivariograma (Perspectiva geométrica I). Esta medida é gerada pela relação entre a área de dependência espacial observada e a área de dependência espacial máxima possível de ser atingida.

A segunda medida tem como base o conceito de que o semivariograma possui duas áreas distintas, sendo uma área de dependência espacial e uma área de não dependência espacial (Perspectiva geométrica II). Esta medida descritiva é construída pela relação entre a área de dependência espacial com a área total do semivariograma (área de dependência espacial + área de não-dependência espacial).

Para a obtenção das medidas são consideradas as duas perspectivas geométricas do semivariograma e, a partir de cada perspectiva, realizados os cálculos das áreas consideradas.

\section{Resultados e Discussão}

No semivariograma é possível definir uma área de dependência espacial que pode indicar o 
tamanho da dependência espacial do fenômeno em estudo. $\mathrm{O}$ desenvolvimento geométrico e de cálculo da área de dependência espacial a partir do semivariograma é apresentado em Seidel (2013) e Seidel e oliveira (2014b). Conceito similar, desenvolvendo uma área de correlação espacial, a partir do correlograma, foi proposto em Seidel (2013) e Seidel e Oliveira (2014a). Neste trabalho é explorado o conceito de área de dependência espacial do semivariograma. A figura 1 mostra um exemplo geral de semivariograma com destaque para a área de dependência espacial.

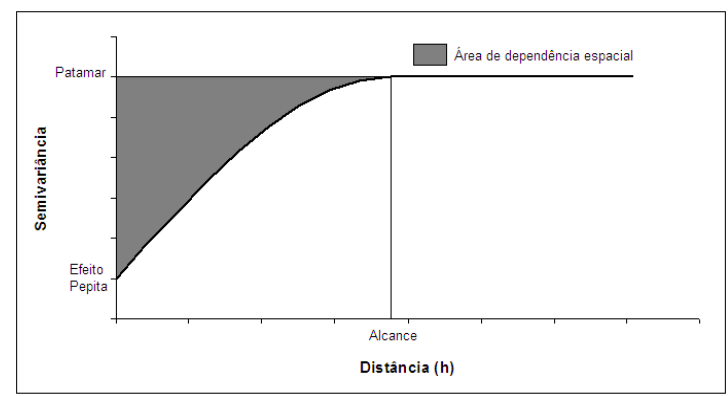

Figura 1 - Representação geral da área de dependência espacial em um semivariograma.

\subsection{Perspectiva geométrica I}

A primeira medida descritiva (índice de dependência espacial), denominada aqui de IDE1, é dada pela razão entre a área de dependência espacial observada e a área de dependência espacial máxima possível de ser atingida. Uma representação dessas duas áreas, para o caso esférico, é dada na figura 2, onde verifica-se que a área de dependência máxima possível de ser atingida é dada por um ajuste esférico iniciando na origem do semivariograma, isto é, tendo efeito pepita igual a zero.

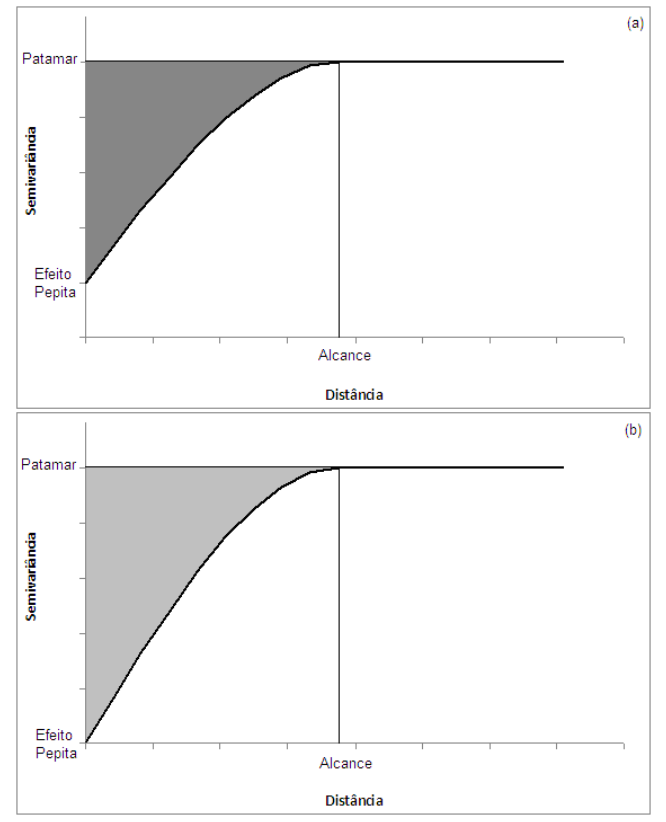

Figura 2 - Área de dependência espacial observada (a) e área de dependência espacial máxima possível de ser atingida (b), no caso esférico.

Como exemplificação, a área de dependência espacial observada ( $\left.A D E_{\text {obs }}\right)$, no caso esférico, é dada por $A D E_{\text {obs }}=\int_{0}^{a}\left(C-\gamma_{\text {esf }}(h)\right) d h$ e a área de dependência espacial máxima possível de ser atingida ( $\left.A D E_{\text {possivel }}\right)$, no caso esférico, é dada por $A D E_{\text {possivel }}=\int_{0}^{a}\left(C-\gamma_{\text {esf }}(h)_{\text {Adaptado }}\right) d h$, em que $C$ é o patamar, $\gamma(h)_{e s f}=C_{0}+C_{1}\left[1,5\left(\frac{h}{a}\right)-0,5\left(\frac{h}{a}\right)^{3}\right] \quad$ e $\gamma_{\text {esf }}(h)_{\text {Adaptado }}=C\left[1,5\left(\frac{h}{a}\right)-0,5\left(\frac{h}{a}\right)^{3}\right]$.

Observa-se que no modelo adaptado $\left(\gamma_{\text {esf }}(h)_{\text {Adaptado }}\right)$, como o efeito pepita é nulo, temse $C_{1}=C$.

Assim, a medida IDE1, no caso esférico, é dada por: 


$$
\begin{aligned}
& I D E 1_{\text {esf }}=\frac{A D E_{\text {obs }}}{A D E_{\text {possivel }}} \\
&= \frac{\int_{0}^{q}\left(C-\gamma_{\text {esf }}(h)\right) d h}{\int_{0}^{q}\left(C-\gamma_{\text {esf }}(h)_{\text {Adaptado }}\right) d h} \\
&= \frac{\int_{0}^{a}\left(C-\left(C_{0}+C_{1}\left[\frac{3}{2}\left(\frac{h}{a}\right)-\frac{1}{2}\left(\frac{h}{a}\right)^{3}\right]\right)\right) d h}{\int_{0}^{a}\left(C-\left(C\left[\frac{3}{2}\left(\frac{h}{a}\right)-\frac{1}{2}\left(\frac{h}{a}\right)^{3}\right]\right)\right) d h} \\
&= \frac{\int_{0}^{a} C d h-\int_{0}^{a} C_{0} d h-\int_{0}^{a} C_{1}\left[\frac{3}{2}\left(\frac{h}{a}\right)-\frac{1}{2}\left(\frac{h}{a}\right)^{3}\right] d h}{\int_{0}^{a} C d h-\int_{0}^{a} C\left[\frac{3}{2}\left(\frac{h}{a}\right)-\frac{1}{2}\left(\frac{h}{a}\right)^{3}\right] d h} \\
&= \frac{C_{1} \cdot a-C_{0} \cdot a-\frac{3}{4} \cdot C_{1} \cdot a+\frac{1}{8} \cdot C_{1} \cdot a}{C \cdot a-\frac{3}{4} \cdot C \cdot a+\frac{1}{8} \cdot C \cdot a} \\
&= \frac{\left[\left(0,375 \cdot\left(C_{1}\right)\right] \cdot a\right.}{[0,375 \cdot(C)] \cdot a} \\
&= \frac{\left(C_{0}+C_{1}\right) \cdot a-C_{0} \cdot a-\frac{3}{4} \cdot C_{1} \cdot a+\frac{1}{8} \cdot C_{1} \cdot a}{C \cdot a-\frac{3}{4} \cdot C \cdot a+\frac{1}{8} \cdot C \cdot a} \\
& {\left[\left(1-\frac{3}{4}+\frac{1}{8}\right) \cdot C_{1}\right] \cdot a } \\
& {\left[C_{0}+C_{1}-C_{0}-\frac{3}{4} \cdot C_{1}+\frac{1}{8} \cdot C_{1}\right] \cdot a }
\end{aligned}
$$

No caso exponencial, o IDE1 é dado por:

$$
\begin{aligned}
& I D E 1_{\exp }=\frac{A D E_{\text {obs }}}{A D E_{\text {possivel }}} \\
& =\frac{\int_{0}^{a}\left(C-\gamma_{\exp }(h)\right) d h}{\int_{0}^{a}\left(C-\gamma_{\exp }(h)_{\text {Adaptado }}\right) d h} \\
& =\frac{\left[0,317 \cdot\left(C_{1}\right)\right] \cdot a}{[0,317 \cdot(C)] \cdot a} \\
& =\frac{C_{1}}{C} \\
& =\frac{C_{1}}{C_{0}+C_{1}}
\end{aligned}
$$

E, no caso gaussiano, tem-se o IDE1 dado por:

$$
\begin{aligned}
& I D E 1_{\text {gaus }}=\frac{A D E_{\text {obs }}}{A D E_{\text {possivel }}} \\
& =\frac{\int_{0}^{a}\left(C-\gamma_{\text {gaus }}(h)\right) d h}{\int_{0}^{a}\left(C-\gamma_{\text {gaus }}(h)_{\text {Adaptado }}\right) d h} \\
& =\frac{\left[0,504 \cdot\left(C_{1}\right)\right] \cdot a}{[0,504 \cdot(C)] \cdot a} \\
& =\frac{C_{1}}{C} \\
& =\frac{C_{1}}{C_{0}+C_{1}}
\end{aligned}
$$

É possível generalizar o IDE1 na forma:

$$
\operatorname{IDE} 1(\%)=\left(\frac{C_{1}}{C_{0}+C_{1}}\right) \cdot 100
$$

em que $C_{0}$ é o efeito pepita e $C_{1}$ é a contribuição.

Pode-se observar que o IDE1 é igual ao índice dado em Biondi, Myers e Avery (1994), ou seja, percebe-se que a expressão do índice (IDE1) derivado das áreas de dependência espacial do semivariograma (esférico, exponencial ou gaussiano) é idêntico ao índice DE.

O IDE1, ao relacionar a área de dependência espacial observada $\left(A D E_{o b s}\right)$ com a área de dependência espacial máxima possível de ser atingida $\left(A D E_{\text {possível }}\right)$, cria uma razão que pode 
assumir valores no intervalo entre 0 e $100 \%$. Assim, a interpretação do $I D E 1$ se refere a quanto da área de dependência espacial possível se atingiu, no fenômeno em estudo, com a área de dependência espacial observada. Além disso, verifica-se que a expressão do $I D E 1$ é a mesma, independentemente do modelo de ajuste considerado (esférico, exponencial ou gaussiano).

No trabalho de Biondi, Myers e Avery (1994), não há nenhuma indicação da forma como foi gerado o índice $\mathrm{DE}$, apenas há a definição de que o DE entende-se como o percentual da variância espacial que é explicada pela dependência espacial. Os autores parecem, apenas, estudar a variabilidade gerada no eixo das semivariâncias no semivariograma, relacionando a variância estruturada (dada pela contribuição) com a variância total (dada pelo patamar).

\subsection{Perspectiva geométrica II}

O segundo índice proposto, denominado de IDE2, é dado pela razão entre a área de dependência espacial observada e a área total observada no semivariograma. Em que, a área total observada é a soma da área de dependência espacial com a área de não-dependência espacial definida até o alcance. Pode-se visualizar, na figura 3, como exemplificação do caso esférico, três áreas destacadas. Uma área de dependência espacial $\left(A D E_{o b s}\right)$, já definida anteriormente, $\mathrm{e}$ duas áreas de não-dependência espacial, ANDE1 e ANDE2. Convenciona-se, neste estudo, conforme Seidel (2013), considerar apenas as áreas $A D E$ e $A N D E 1$, definidas até o alcance, que somadas resultam na área de dependência espacial total $\left(A T_{o b s}\right)$. A área total é dada como $A T_{o b s}=A D E_{o b s}+A N D E 1_{o b s}=C . a$, em que $C$ é o patamar e $a$ é o alcance prático.

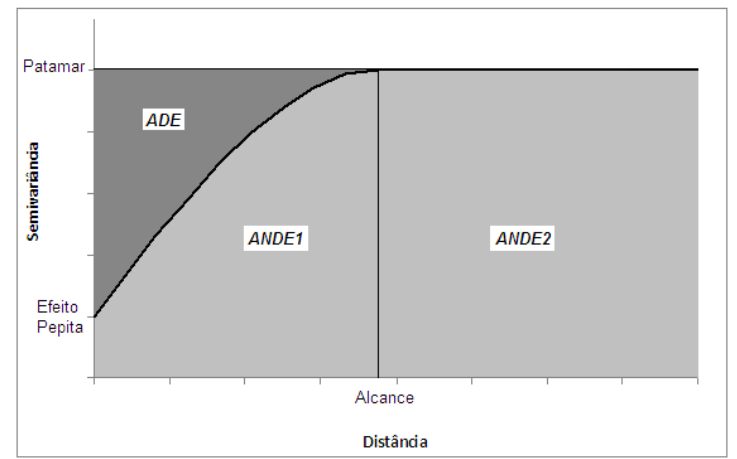

Figura 3 - Áreas de dependência e nãodependência espaciais, no caso esférico.

Assim, a medida IDE2, no caso esférico, é dada por:

$I D E 2_{e s f}=\frac{A D E_{o b s}}{A T_{o b s}}$

$=\frac{\int_{0}^{a}\left(C-\gamma_{e s f}(h)\right) d h}{C . a}$

$=\frac{\left[0,375 \cdot\left(C_{1}\right)\right] \cdot a}{C \cdot a}$

$=\frac{0,375 \cdot\left(C_{1}\right)}{C}$

$=\frac{0,375 \cdot\left(C_{1}\right)}{C_{0}+C_{1}}$

No caso exponencial, o IDE2 é dado por:

$$
\begin{aligned}
& I D E 2_{\exp }=\frac{A D E_{o b s}}{A T_{o b s}} \\
& =\frac{\int_{0}^{a}\left(C-\gamma_{\exp }(h)\right) d h}{C \cdot a} \\
& =\frac{\left[0,317 \cdot\left(C_{1}\right)\right] \cdot a}{C \cdot a} \\
& =\frac{0,317 \cdot\left(C_{1}\right)}{C} \\
& =\frac{0,317 \cdot\left(C_{1}\right)}{C_{0}+C_{1}}
\end{aligned}
$$

E, no caso gaussiano, tem-se o IDE2 dado por: 


$$
\begin{aligned}
& I D E 2_{\text {gaus }}=\frac{A D E_{\text {obs }}}{A T_{\text {obs }}} \\
& =\frac{\int_{0}^{a}\left(C-\gamma_{\text {gaus }}(h)\right) d h}{C \cdot a} \\
& =\frac{\left[0,504 \cdot\left(C_{1}\right)\right] \cdot a}{C \cdot a} \\
& =\frac{0,504 \cdot\left(C_{1}\right)}{C} \\
& =\frac{0,504 \cdot\left(C_{1}\right)}{C_{0}+C_{1}}
\end{aligned}
$$

É possível generalizar o IDE2 na forma:

$$
\operatorname{IDE} 2(\%)=F M \cdot\left(\frac{C_{1}}{C_{0}+C_{1}}\right) \cdot 100,
$$

em que $F M$ é o fator de modelo, $C_{0}$ é o efeito pepita e $C_{1}$ é a contribuição. No caso esférico tem-se $\mathrm{FM}=0,375$. No caso exponencial tem-se $\mathrm{FM}=0,317$. $\mathrm{E}$, no caso gaussiano tem-se $\mathrm{FM}=0,504$.

Assim, é possível definir o IDE2 para os casos esférico, exponencial e gaussiano, respectivamente, por:

$$
\begin{aligned}
& I D E 2_{\text {esf }}(\%)=0,375 .\left(\frac{C_{1}}{C_{0}+C_{1}}\right) \cdot 100, \\
& I D E 2_{\exp }(\%)=0,317 .\left(\frac{C_{1}}{C_{0}+C_{1}}\right) \cdot 100,
\end{aligned}
$$

e

$$
I D E 2_{\text {gaus }}(\%)=0,504 .\left(\frac{C_{1}}{C_{0}+C_{1}}\right) \cdot 100 \text {, }
$$

em que $C_{0}$ é o efeito pepita e $C_{1}$ é a contribuição.

O IDE2, no caso esférico, varia de 0 a $37,5 \%$; no caso exponencial, varia de 0 a $31,7 \%$; e no caso gaussiano, varia no intervalo de 0 a 50,4\%. Esta amplitude de valores é idêntica à do IDE proposto por Seidel e Oliveira (2014a).

Fazendo uma analogia com o índice DE apresentado em Biondi, Myers e Avery (1994) é possível criar uma escala de classificação para o
IDE2 de modo a tornar mais fácil sua interpretação. Por exemplo, o DE tem a seguinte classificação, adaptada de Cambardella et al. (1994):

$\mathrm{DE}(\%) \leq 25 \% \Rightarrow$ Fraca dependência espacial;

$25 \%<\mathrm{DE}(\%) \leq 75 \%=>$ Moderada dependência;

$\mathrm{DE}(\%)>75 \%=>$ Forte dependência espacial.

Assim, o IDE2, no modelo esférico, tem a seguinte classificação:

IDE2(\%) $\leq 9 \%$ $\Rightarrow$ Fraca dependência espacial;

$9 \%<$ IDE2 $(\%) \leq 28 \%=>$ Moderada dependência;

IDE2(\%) $>28 \%$ => Forte dependência espacial.

No modelo exponencial, tem a seguinte classificação:

IDE2 $(\%) \leq 8 \%$ => Fraca dependência espacial;

$8 \%<\operatorname{IDE} 2(\%) \leq 24 \%$ => Moderada dependência;

IDE2(\%) $>24 \% \Rightarrow$ Forte dependência espacial.

E, no modelo gaussiano, tem a seguinte classificação:

IDE2 $(\%) \leq 13 \%$ => Fraca dependência espacial;

$13<$ IDE2 $(\%) \leq 38 \%$ => Moderada dependência;

IDE2 $(\%)>38 \%=>$ Forte dependência espacial.

O índice IDE2 parece descrever de forma mais adequada a dependência espacial dos fenômenos, em relação ao IDE1, já que, representa o percentual da área total (soma entre as áreas de dependência e não-dependência espaciais) do semivariograma, que é ocupada pela área de dependência espacial.

\section{Conclusão}

O IDE1 é idêntico ao índice apresentado em Biondi, Myers e Avery (1994), ou seja, é possível dizer que encontrou-se, a partir da geometria do semivariograma, a demonstração matemática do índice DE.

Contudo, deve-se observar que esse índice apresenta a fragilidade de só considerar os parâmetros contribuição e efeito pepita, e não o alcance e/ou o modelo ajustado. 
O IDE2 é mais completo que o índice IDE1, pois considera o fator de modelo, mostrando que há diferenças na dependência espacial dos fenômenos a partir dos modelos que os descrevem.

Contudo, esse índice também apresenta a fragilidade de só considerar, além do modelo ajustado, a contribuição e o efeito pepita em sua composição, não contemplando o alcance.

Neste sentido, pode-se considerar que as medidas IDE1 e IDE2 podem ser usadas para descrever a dependência espacial, mas devem ser usados em conjunto com outras medidas que contemplem o alcance de dependência espacial, como é o caso do índice IDE proposto por Seidel e Oliveira (2014a).

\section{Referências}

BIONDI, F.; MYERS, D. E.; AVERY, C. C. Geostatistically modeling stem size and increment in an old-growth forest. Canadian Journal of Forest Research, Ottawa, v. 24, n. 7, p. 1354-1368, July 1994.

CAMBARDELLA, C.A.; MOORMAN, T.B.; NOVAK, J.M.; PARKIN, T.B.; KARLEN, D.L.; TURCO, R.F. \& KONOPKA, A.E. Field-scale variability of soil properties in Central Iowa soils. Soil Science Society America Journal, Madison, v. 58, n. 5, p. 1501-1511, Sept./Oct. 1994.

CARVALHO, J. R. P.; SILVEIRA, P. M.; VIEIRA, S. R. Geoestatística na determinação da variabilidade espacial de características químicas do solo sob diferentes preparos. Pesquisa Agropecuária Brasileira, Brasília, v. 37, n. 8, p. 1151-1159, ago. 2002. Disponível em: $<$ http://www.scielo.br/scielo.php?script=sci_artte xt\&pid=S0100-

204X2002000800013\&lng=en\&nrm=iso/v37n8/\&tl ng=pt $>$. Acesso em: 18 abr. 2015. doi: 10.1590/S0100-204X2002000800013.

ISAAKS, E. H.; SRIVASTAVA, R. M. Applied geostatistics. New York: Oxford University, 1989. $561 \mathrm{p}$.

OLIVEIRA, M. C. N.; BARBIN, D.; SILVA, E. F.; MACEDO, J. R.; CAPECHE, C. L.; HISSA, H. R. Estimação dos parâmetros de uma função de covariância no estudo da variabilidade espacial do teor de cálcio no solo. Revista de Matemática e Estatística, Marília, v. 24, n. 4, p. 117-132, 2006. Disponível em: $<$ http://jaguar.fcav.unesp.br/RME/fasciculos/v24/ v24 n4/A8 Cristina.pdf $>$. Acesso em: 02 abr. 2015.

SEIDEL, E. J. Novas contribuições para avaliação e descrição da estrutura de dependência espacial em Geoestatística. Lavras, 2013. 146 p. Tese (Doutorado em Estatística e Experimentação Agropecuária), Universidade Federal de Lavras, Lavras, 2013.

SEIDEL, E. J.; OLIVEIRA, M. S. Proposta de uma generalização para os modelos de semivariogramas exponencial e gaussiano. Semina: Ciências Exatas e Tecnológicas, v. 34, n. 1, p. 125-132, 2013. Disponível em: $<$ http://www.uel.br/revistas/uel/index.php/seme xatas/article/view/14114>. Acesso em: 02 abr. 2015. doi: 10.5433/1679-0375.2013v34n1p125.

SEIDEL, E. J.; OLIVEIRA, M. S. Novo índice geoestatístico para a mensuração da dependência espacial. Revista Brasileira de Ciência do Solo, v. 38, n. 3, p. 699-705, 2014a. Disponível em: <http://www.scielo.br/scielo. php?script=sci arttext\&pid=S0100-

$06832014000300002 \& \operatorname{lng}=$ pt\&nrm $=$ iso $>$. Acesso em: 20 jul. 2014 doi: 10.1590/S010006832014000300002 .

SEIDEL, E. J.; OLIVEIRA, M. S. Proposta de um teste de hipótese para a existência de dependência espacial em dados geoestatísticos. Boletim de Ciências Geodésicas, v. 20, n. 4, p. 750-764, 2014b. Disponível em: $<$ http://www.scielo.br/scielo.php?script=sci artte xt\&pid=S1982-

21702014000400750\&lng $=$ en\&nrm $=$ iso $>$. Acesso em: 18 abr. 2015. doi: 10.1590/S198221702014000400043.

SILVA, F. M.; ALVES, M. C.; SOUZA, J. C. S.; OLIVEIRA, M. S. Efeitos da colheita manual na bienalidade do cafeeiro em Ijaci, Minas Gerais. Ciência e Agrotecnologia, Lavras, v. 34, n. 3, p. 625-632, maio/jun. 2010. Disponível em: $<$ http://www.scielo.br/scielo.php?script=sci artte xt\&pid=S1413-

70542010000300014\&lng=pt\&nrm=iso\&tlng $=$ pt $>$.

Acesso em: 18 abr. 2015. doi: 10.1590/S141370542010000300014. 